\title{
Characterization of iron metabolism and erythropoiesis in erythrocyte membrane defects and thalassemia traits
}

\author{
Lucie Sulovska a\#, Dusan Holub ${ }^{\text {b\# }}$, Zuzana Zidovac, Martina Divokad, Marian Hajduch ${ }^{b}$, Vladimir Mihala , Jana Vrbkovab, \\ Monika Horvathovac, Dagmar Pospisilova ${ }^{a}$
}

Background and Aims. Erythropoiesis is closely related to iron metabolism in a balanced homeostasis. Analyses of diverse erythroid and iron metabolism disorders have shown that disrupted erythropoiesis negatively affects iron homeostasis and vice versa. The aim of this study was to characterize the relationship between erythropoietic activity and iron homeostasis in pediatric patients with erythrocyte membrane defects and thalassemia traits.

Methods. Selected markers of erythropoietic activity (erythropoietin, soluble transferrin receptor - sTfR and growth differentiation factor 15) and iron status parameters (serum iron, ferritin and hepcidin) were evaluated in pediatric patients with erythrocyte membrane defects and thalassemia traits.

Results. The patients with erythrocyte membrane defects and thalassemia traits had altered iron homeostasis due to disturbed erythropoiesis. In comparison with healthy controls, they had a normal to low hepcidin/ferritin ratio and concomitantly elevated sTfR.

Conclusion. The findings suggest that pediatric patients with erythrocyte membrane defects and thalassemia traits are more susceptible to iron overload than the general population and that the (hepcidin/ferritin)/sTfR ratio can be used to monitor any worsening of the disease.

Key words: hepcidin, erythropoietic activity, erythrocyte membrane defects, thalassemia trait

Received: June 15, 2015; Accepted with revision: October 16, 2015; Available online: October 27, 2015

http://dx.doi.org/10.5507/bp.2015.054

${ }^{a}$ Department of Pediatrics, Faculty of Medicine and Dentistry, Palacky University Olomouc and University Hospital Olomouc, Czech Republic bInstitute of Molecular and Translational Medicine, Faculty of Medicine and Dentistry, Palacky University Olomouc, Czech Republic 'Department of Biology, Faculty of Medicine and Dentistry, Palacky University Olomouc, Czech Republic

${ }^{d}$ Department of Hemato-oncology, Faculty of Medicine and Dentistry, Palacky University Olomouc and University Hospital Olomouc, Czech Republic

"The authors contributed equally to this manuscript

Corresponding author: Monika Horvathova, e-mail: monika.horvathova@upol.cz

\section{INTRODUCTION}

Anemia, one of the most common pathological conditions of childhood, can adversely affect development owing to inappropriate oxygen supply to developing organs and tissues. The most frequent cause is nutritional iron deficiency known as iron deficiency anemia (IDA) which results in hypochromic and microcytic red blood cells. IDA however must be distinguished from inherited microcytic anemias, especially $\beta$-thalassemia, which is characterized by imbalanced production of globin chains. Anemia can also be associated with excessive destruction of red blood cells (hemolysis); the most common causes of inherited hemolytic anemia in the Czech population are erythrocyte membrane defects, predominantly hereditary spherocytosis.

\section{Thalassemia}

Thalassemias are a heterogeneous group of inherited disorders characterized by reduced or absent synthesis of one or several globin chains which are a part of the hemoglobin $(\mathrm{Hb})$ molecule ${ }^{1}$. Under physiological conditions, the ratio of $\alpha$-and non- $\alpha$-globin chains (i.e. predominantly $\beta$-globins in adults) in red blood cells is balanced (1:1).
A reduced amount of $\alpha$ - or $\beta$-globin chain produced by a mutant allele is referred as $\alpha^{+}$or $\beta^{+}$thalassemia while a total absence of $\alpha$ - or $\beta$-globin chain production is called $\alpha^{0}$ or $\beta^{0}$ thalassemia. The pathophysiology and clinical symptoms of thalassemia are related to the extent of imbalance between $\alpha$ - and $\beta$-globin chain synthesis. Heterozygous carriers of a $\beta$-thalassemia allele are usually asymptomatic, with hypochromic and microcytic erythrocytes, mild anemia accompanied by compensatory increase in the number of red blood cells (erytrocytosis) and slightly increased erythropoiesis in bone marrow. This type of thalassemia is known as $\beta$-thalassemia minor or $\beta$-thalassemia trait and represents the most common cause of hereditary microcytic anemia in the Czech Republic. More profound imbalance in $\alpha$ - and $\beta$-globin chain synthesis leads to $\beta$-thalassemia intermedia; patients exhibit moderate anemia with signs of hemolysis, ineffective erythropoiesis and splenomegaly. Some patients may require occasional transfusions. The incidence of $\beta$-thalassemia intermedia in the Czech Republic is very low. The most severe form of $\beta$-thalassemia is $\beta$-thalassemia major. $\mathrm{Hb}$ levels at diagnosis may be below $3.0 \mathrm{~g} / \mathrm{dL}$ (normal range: $12.0-15.0 \mathrm{~g} / \mathrm{dL}$ ) and patients require regular transfusion therapy ${ }^{1}$. To date, no cases of $\beta$-thalassemia major have 
been reported in the Czech population but sporadic cases have been diagnosed in immigrants. The clinical picture of $\alpha$-thalassemia is dependent on the degree of reduction of $\alpha$-globin chain synthesis; i.e. on total number of mutated $\alpha$-globin genes. Four $\alpha$-globin genes are present in the normal diploid human genome. Individuals with deletion/ inactivation of one allele are asymptomatic and considered silent carriers; the incidence in the Czech Republic may be relatively high. Individuals who inherit two mutant alleles are usually clinically asymptomatic with mild hypochromic microcytic anemia and slightly increased red blood cell count. This condition, called $\alpha$-thalassemia minor or $\alpha$-thalassemia trait, is relatively rare in the Czech Republic (M. Divoka, unpublished data). Hemoglobin $\mathrm{H}$ disease results from mutation affecting three $\alpha$-globin genes and presents with moderate to severe anemia. Sporadic cases may be found in the Czech population. The inactivation of all four $\alpha$-globin genes is lethal and leads to hydrops fetalis and fetal death ${ }^{2}$.

\section{Erythrocyte membrane defects}

Hereditary defects of erythrocyte membrane proteins manifest as clinically and genetically heterogeneous disorders. The result is large numbers of abnormally shaped erythrocytes, i.e. spherical (for hereditary spherocytosis - HS) or elliptical (for hereditary elliptocytosis - HE) rather than the normal biconcave disk shape ${ }^{3}$. These abnormal erythrocytes have a shorter than average life-span for normal red blood cells and die prematurely. This leads to a compensatory increase in reticulocyte count which is more pronounced in HS than HE. The patient may be asymptomatic or present with varying degree of hemolytic anemia, increased serum bilirubin and splenomegaly. Transfusion therapy for the most severe cases of HS and HE may lead to iron overload which can cause severe health problems ${ }^{3}$.

\section{Hepcidin and iron metabolism}

Erythropoiesis and iron metabolism reciprocate in the coordinated supply of iron and globin synthesis, essential for normal red blood cell production. Analyses of diverse erythroid and iron metabolism disorders have shown that disrupted erythropoiesis negatively affects iron homeostasis and vice versa.

Iron $(\mathrm{Fe})$ is vital for a number of metabolic processes, most importantly, oxygen transport as a part of the $\mathrm{Hb}$ molecule. However, because of its potential toxicity, iron levels must be strictly regulated. Hepcidin, the key molecule regulating plasma iron levels, is produced by hepatocytes ${ }^{4}$. Hepcidin binds to an iron exporter, ferroportin expressed in the cytoplasmic membrane. This induces ferroportin internalization and degradation, resulting in blocking iron release from sites of iron absorption (duodenal enterocytes), iron recycling (macrophages) and iron storage (hepatocytes). Hepcidin synthesis is stimulated in response to increased iron stores and inflammation. It is attenuated in response to iron deficiency and accelerated erythropoiesis due to anemia, ineffective erythropoiesis and hypoxia 5 . The molecules that induce hepcidin synthesis in response to increased iron levels and inflamma- tion are relatively well- defined ${ }^{4,5}$. On the other hand, the identity of negative regulators of hepcidin is still elusive. Growth differentiation factor 15 (GDF15) was proposed as a candidate molecule in the suppression of hepcidin production in patients with $\beta$-thalassemia ${ }^{6}$. Later analyses showed that GDF15 was elevated in disease states associated with ineffective erythropoiesis ${ }^{7}$. More recent data indicate the existence of other negative erythroid regulators of hepcidin synthesis ${ }^{8}$. It is accepted that these signals are released by erythroid precursors in bone marrow ${ }^{9}$ and override the signals of iron stores ${ }^{5}$.

Imbalances in iron metabolism (including hepcidin levels) and their interconnection with defective erythropoiesis have been widely studied in $\beta$-thalassemia (intermedia and major) (ref. ${ }^{10}$ ), congenital dyserythropoietic anemia $^{11}$, pyruvate kinase deficiency ${ }^{12}$ and DiamondBlackfan anemia ${ }^{13}$. In this study, we investigated the relationship between iron metabolism and erythropoietic activity in pediatric patients with erythrocyte membrane defects and thalassemia traits.

\section{MATERIALS AND METHODS}

\section{Characterization of the cohort}

The cohort consisted of 20 patients with erythrocyte membrane defect ( 18 with HS and 2 with HE) (age range 2-18 years), 13 with thalassemia minor: 10 with the $\beta$-thalassemia trait and 3 with the $\alpha$-thalassemia trait (age range 3-17 years) and 1 patient with thalassemia major (TM) (age 7 years), an immigrant from Moldova. Thalassemia carriers did not require any treatment. The patient with TM was on regular transfusion therapy (1 transfusion unit every three weeks). A control group included 47 children examined prior to planned minor surgery (inguinal or umbilical hernia, plastic surgery). The study and informed consent as per the Declaration of Helsinki were approved by the Ethics Committee of Palacky University Hospital, Olomouc, Czech Republic. None of the subjects had signs of an inflammatory process at the time of examination as documented by normal C-reactive protein (CRP) levels (not shown).

\section{Hematological and biochemical analysis}

Blood was taken during routinely performed venous puncture; for the TM patient a pre-transfusion sample was collected. Blood counts and erythrocyte characteristics were examined on Sysmex XE-500 analyzer (Sysmex) for all subjects included in the study. Biochemical serum parameters of iron metabolism and inflammation: serum iron (Fe), ferritin, transferrin saturation (TSAT), soluble transferrin receptor (sTfR) and CRP levels were determined using standard methods; for the control group, samples from 10 to 13 children were analyzed. The serum erythropoietin (EPO) concentration was measured by a solid-phase chemiluminescent immunochemical reaction $^{14}$. Laboratory reference values are given for EPO instead of direct control group measurements.

The diagnosis of HS and HE was made on the basis of elevated bilirubin levels (mean $29 \mu \mathrm{mol} / \mathrm{L}$, patients range 
8-70 $\mu \mathrm{mol} / \mathrm{L}$, normal range 5-21 $\mu \mathrm{mol} / \mathrm{L})$, positive results of cryohemolysis test and blood smear assessment. Thalassemias were diagnosed on the basis of hemoglobin electrophoresis and globin gene Sanger sequencing ${ }^{15}$.

\section{Hepcidin analysis}

The serum hepcidin levels were measured by reversephase liquid chromatography using the UltiMate 3000 Nano LC System (Thermo Fisher Scientific, Sunnyvale, CA, USA) coupled to the QTRAP 5500 mass spectrometer (AB SCIEX, Framingham, MA, USA) as we previously described ${ }^{12}$. Hepcidin was determined for 47 healthy controls, 12 thalassemia carriers and 20 patients with erythrocyte membrane defect.

\section{GDF15 measurements}

The serum levels of growth differentiation factor 15 (GDF15) were quantified according to the manufacturer's instructions using the Human GDF15 Quantikine ELISA Kit (R\&D Systems, Inc., Minneapolis, MN, USA). GDF15 measurements were performed on 9 thalassemia carriers and 11 patients with erythrocyte membrane defect.

\section{Statistical analysis}

ANOVA and Dunnett's test for normally distributed variables or non-parametric Kruskal-Wallis test with multiple comparisons were used for the statistical evaluation. All statistical analyses were done using the STATISTICA, (StatSoft, Inc., software version 12). The significance level was set at $5 \%$ for all analyses.

\section{RESULTS}

\section{Hematological data and erythropoietic activity}

We first analyzed the hematological data. Carriers of $\alpha$ - thalassemia were evaluated together with $\beta$-thalassemia carriers due to similarities in clinical picture. They showed mild to severe anemia with a compensatory increase in RBC counts (Table 1). Hemoglobin ( $\mathrm{Hb}$ ), mean corpuscular volume (MCV), mean corpuscular hemoglobin $(\mathrm{MCH})$ and mean corpuscular hemoglobin concentration (MCHC) were significantly lower compared to healthy controls. In the erythrocyte membrane defect group, the anemia was milder, with significantly reduced $\mathrm{RBC}$ count

Table 1. Hematological parameters.

\begin{tabular}{lcccc}
\hline & Controls & Thalassemia carriers & $\begin{array}{c}\text { Erythrocyte } \\
\text { membrane defects }\end{array}$ & Thalassemia major \\
\hline $\mathrm{RBC}\left(10^{12} / \mathrm{L}\right)$ & $4.78 \pm 0.60$ & $5.55^{* * *} \pm 0.315$ & $4.23^{*} \pm 0.71$ & 2.97 \\
$\mathrm{Hb}(\mathrm{g} / \mathrm{dL})$ & $13.0 \pm 1.3$ & $10.6^{* * * *} \pm 1.1$ & $12.0 \pm 1.9$ & 8.2 \\
$\mathrm{MCV}(\mathrm{fL})$ & $82.56 \pm 6.87$ & $59.1^{* * *} \pm 5.68$ & $79.1 \pm 5.73$ & 79.8 \\
$\mathrm{MCH}(\mathrm{pg})$ & $28.29 \pm 2.62$ & $19.23^{* * * *} \pm 2.27$ & $28.45 \pm 1.86$ & 27.6 \\
$\mathrm{MCHC}(\mathrm{g} / \mathrm{dL})$ & $34.32 \pm 1.22$ & $32.46^{*} \pm 1.21$ & $36.09^{* * *} \pm 0.87$ & 34.6 \\
Ret (ratio) & $0.010 \pm 0.003$ & $0.012 \pm 0.004$ & $0.066^{* * *} \pm 0.045$ & 0.012 \\
\hline
\end{tabular}

Values are shown as means $\pm \mathrm{SD}$; single values are given for the patient with thalassemia major. RBC, red blood cells count; Hb, hemoglobin; $\mathrm{MCV}$, mean corpuscular volume; $\mathrm{MCH}$, mean corpuscular hemoglobin; $\mathrm{MCHC}$, mean corpuscular hemoglobin concentration, Ret, reticulocytes count. Statistical significance relative to control group: ${ }^{*} P$ value $<0.05 ;{ }^{* *} P$ value $<0.01 ;{ }^{* * *} P$ value $<0.001$.

Table 2. Parameters of erythropoietic activity.

\begin{tabular}{|c|c|c|c|c|}
\hline & Controls & $\begin{array}{c}\text { Thalassemia } \\
\text { carriers }\end{array}$ & $\begin{array}{c}\text { Erythrocyte } \\
\text { membrane defects }\end{array}$ & $\begin{array}{c}\text { Thalassemia } \\
\text { major }\end{array}$ \\
\hline sTfR (mg/L) & $3.7 \pm 0.74$ & $6.89 \pm 5.02$ & $9.58^{* *} \pm 4.89$ & 19 \\
\hline EPO (IU/L) & {$[4.3-29]^{+}$} & $18.0 \pm 22.67$ & $34.04 \pm 30.45$ & $>4000$ \\
\hline GDF $15(\mathrm{pg} / \mathrm{mL})$ & $205 \pm 27$ & $653^{* *} \pm 545$ & $387 \pm 131$ & 8316.7 \\
\hline
\end{tabular}

Values are shown as means \pm SD; single values are given for the patient with thalassemia major. sTfR, soluble transferrin receptor; EPO, erythropoietin; GDF 15, growth differentiation factor 15 ; + reference range. Statistical significance relative to control group: **P value<0.01.

Table 3. Iron status parameters.

\begin{tabular}{lccrr}
\hline & Controls & $\begin{array}{c}\text { Thalassemia } \\
\text { carriers }\end{array}$ & $\begin{array}{c}\text { Erythrocyte } \\
\text { membrane defects }\end{array}$ & $\begin{array}{c}\text { Thalassemia } \\
\text { major }\end{array}$ \\
\hline \%TSAT & $33.07 \pm 14.09$ & $30.9 \pm 10.86$ & $29.05 \pm 10.22$ & 59.9 \\
Serum Fe $(\mu \mathrm{M} / \mathrm{L})$ & $20.87 \pm 8.37$ & $16.75 \pm 5.57$ & $15.04 \pm 4.57$ & 22 \\
Ferritin $(\mu \mathrm{g} / \mathrm{L})$ & $34.62 \pm 20.1$ & $40.23 \pm 39.15$ & $99.54^{* *} \pm 45.52$ & 2872 \\
Hepcidin $(\mathrm{ng} / \mathrm{mL})$ & $25.86 \pm 26.46$ & $14.73 \pm 14.27$ & $15.34 \pm 16.56$ & 56.2 \\
Hepcidin/ferritin & $0.658 \pm 0.564$ & $0.341 \pm 0.149$ & $0.177^{* *} \pm 0.173$ & 0.02 \\
(hepcidin/ferritin)/sTfR & $0.184 \pm 0.189$ & $0.072 \pm 0.050$ & $0.023^{* * *} \pm 0.021$ & 0.001 \\
\hline
\end{tabular}

Values are shown as means \pm SD; single values are given for the patient with thalassemia major. TSAT, transferrin saturation; Fe, iron; sTfR, soluble transferrin receptor. Statistical significance relative to control group: ${ }^{*} P$ value $<0.01,{ }^{* * *} P$ value $<0.001$. 

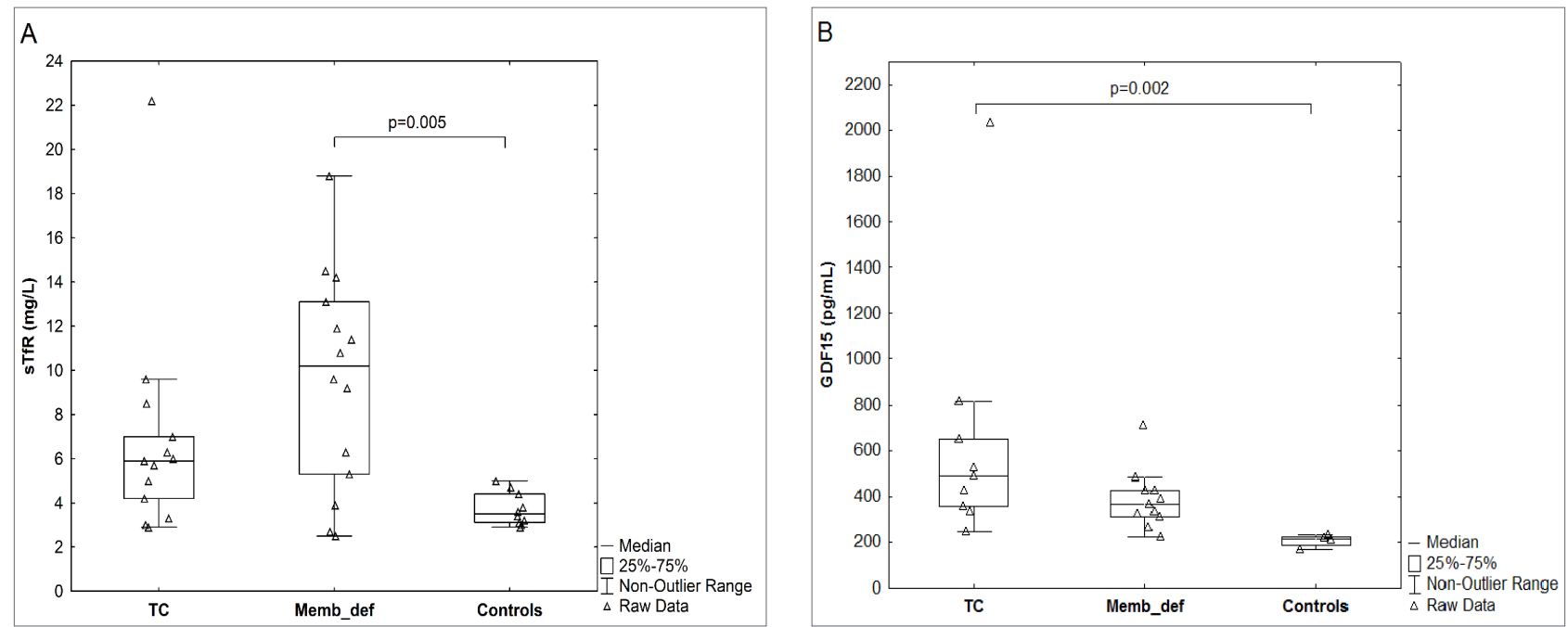

Fig. 1. Elevated markers of erythropoietic activity. (A) sTfR was increased in both patients' groups when compared to the controls $(n=9)$, but only in patients with erythrocyte membrane defect (Memb_def; $n=14)$ the difference reached statistical significance; thalassemia carriers (TC, $n=13)$. (B) The elevation of GDF15 in comparison to the controls was significant for TC ( $n=9)$, but not for Memb_def $(n=11)$. sTfR, soluble transferrin receptor; GDF15, growth differentiation factor 15. The graphs show individual values (triangles), 25-75\% range with median (box and horizontal line, respectively) and the non-outlier range (the highest and the lowest whiskers, respectively).

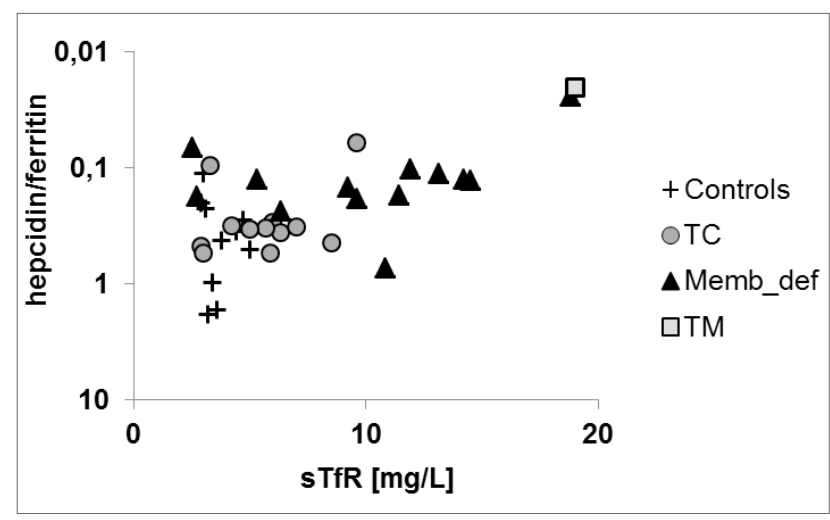

Fig. 2. Differentiation of patients based on the plot of hepcidin/ ferritin ratio against $\mathrm{STfR}^{15}$. Patients with erythrocyte membrane defect (Memb_def, $n=13$ ), thalassemia carriers (TC, $n=12$ ) and thalassemia major (TM, $\mathrm{n}=1)$ dislocate from healthy controls $(n=9)$.

and moderate changes in RBC characteristics (Table 1) with the exception of $\mathrm{MCHC}$, which was significantly increased. In addition, compensatory reticulocytosis in response to chronic hemolysis was observed in the majority of patients with erythrocyte membrane defect causing the number of reticulocytes to be significantly different from the control group (Table 1).

We next examined the markers of erythropoietic activity, i.e. serum erythropoietin (EPO) and soluble transferrin receptor (sTfR). EPO was elevated above the reference range (4.3 - $29 \mathrm{IU} / \mathrm{L})$ only in the group of erythrocyte membrane defects (mean 34 IU/L, Table 2); in thalassemia carriers the mean value fell within the reference range (mean 18.0 IU/L, Table 2). sTfR was also significantly higher for patients with erythrocyte membrane defect (mean $9.58 \mathrm{mg} / \mathrm{L}$ ) than in healthy controls $(3.7$ $\mathrm{mg} / \mathrm{L}$, Table 2 and Fig. 1A). In the thalassemia carri- ers' group mean sTfR $(6.89 \mathrm{mg} / \mathrm{L})$ was 1.9 times higher than mean sTfR of the controls (Table 2 and Fig. 1A). Although the difference was not statistically significant, 10 out of 13 thalassemia carriers had sTfR above the mean control value (Fig. 1A). This indicates increased erythropoietic activity in the bone marrow of thalassemia carriers and patients with erythrocyte membrane defect. To assess the degree of ineffective erythropoiesis ${ }^{16,17}$, serum growth differentiation factor 15 (GDF15) was measured using ELISA. The mean GDF15 level was increased in both groups (thalassemia carriers, $653 \mathrm{pg} / \mathrm{mL}$; erythrocyte membrane defects, $387 \mathrm{pg} / \mathrm{mL}$ ) compared to the controls $(205 \mathrm{pg} / \mathrm{mL})$, but only in thalassemia carriers did the difference reach statistical significance (Table 2 and Fig. 1B).

Our only patient with thalassemia major (TM), who is a Moldovan immigrant living in the Czech Republic, 
presented with the most severe anemia ( $\mathrm{Hb}$ of $8.2 \mathrm{~g} / \mathrm{dL})$, reduced RBC number, elevated sTfR and EPO levels and the highest GDF15 (Table 1 and 2).

\section{Iron status and hepcidin}

To assess any disturbance in iron homeostasis, selected parameters of iron metabolism were evaluated. As shown in Table 3, serum iron ( $\mathrm{sFe}$ ) and transferrin saturation (TSAT) were comparable in all groups. Patients with erythrocyte membrane defect had significantly increased levels of serum ferritin (mean $99.54 \mu \mathrm{g} / \mathrm{L}$; mean for controls, $34.62 \mu \mathrm{g} / \mathrm{L}$ ). Ferritin levels of thalassemia carriers did not differ from healthy controls (Table 3 ). Hepcidin levels were reduced in thalassemia carriers (mean 14.73 $\mathrm{ng} / \mathrm{mL}$ ) and erythrocyte membrane defects (mean 15.34 $\mathrm{ng} / \mathrm{mL}$ ) compared to controls (mean $25.86 \mathrm{ng} / \mathrm{mL}$, Table 3 ), but this was not statistically significant. We therefore calculated the hepcidin/ferritin ratio which represents a measure of appropriate response of hepcidin to iron stores. As shown in Table 3, this ratio was significantly lower in the erythrocyte membrane defects group (0.177) compared to healthy individuals $(0.658)$. The reduction of hepcidin/ferritin ratio in thalassemia carriers (to 0.341 ) reached only borderline statistical significance; even though it was twice as low as in the controls. This indicates inappropriate suppression of hepcidin synthesis.

Along with the changes in hematological parameters, alterations in iron status in our TM patient was consistent with the data published on large cohorts of TM patients ${ }^{10,18,19}$. She showed normal $\mathrm{sFe}$, but significant hyperferritinemia (ferritin level of $2872 \mu \mathrm{g} / \mathrm{L}$, Table 3 ). Her serum hepcidin was twice as high $(56.2 \mathrm{ng} / \mathrm{mL})$ as in healthy controls $(25.86 \mathrm{ng} / \mathrm{mL})$. However, the hepcidin/ ferritin ratio, which was 0.02 (control hepcidin/ferritin ratio is 0.658 ) indicated that hepcidin was disproportionally low with respect to iron loading (Table 3 ).

Finally, we calculated the ratio of (hepcidin/ferritin) to sTfR which combines the parameters of iron status and erythropoietic activity. Recently, it has been suggested that this formula distinguishes adult thalassemia carriers from healthy controls and reflects the severity of the ane$\mathrm{mia}^{20}$. As shown in Table 3 , this ratio is reduced in both thalassemia carrier group (0.072) and erythrocyte membrane defects $(0.023)$ compared to healthy individuals (0.184) confirming disordered interaction between iron metabolism and erythropoiesis. When sTfR is plotted against the hepcidin/ferritin ratio, thalassemia carriers and patients with erythrocyte membrane defect, tend to diverge from controls (Fig. 2); showing slight to moderate reduction in hepcidin/ferritin ratio and concomitant increase in sTfR that is consistent with accelerated bone marrow erythropoiesis. The TM patient had the lowest (hepcidin/ferritin)/sTfR ratio (0.001) and can be found in the upper right corner on the graph plotting sTfR against hepcidin/ferritin with the lowest hepcidin/ferritin and highest sTfR (Fig. 2). This result confirms increased bone marrow erythropoiesis and inadequate suppression of hepcidin to the degree of iron loading.

\section{DISCUSSION}

A number of congenital anemias are coupled with disrupted iron balance and secondary iron overload. The molecular pathophysiology involves suppression of hepcidin; the key molecule inhibiting iron absorption and recycling ${ }^{21}$. An important finding is that erythroid signals dominate over iron store signals ${ }^{5}$. As a result, patients with ineffective erythropoiesis and concomitantly increased iron levels have inappropriately low hepcidin with respect to iron loading which leads to the accumulation of excessive iron in parenchymal tissues. These relatively suppressed hepcidin levels, explain iron overload in patients with $\beta$-thalassemia intermedia that are transfusion independent ${ }^{18}$. On the other hand, the primary cause of iron overload in $\beta$-thalassemia major is regular transfusion therapy; hepcidin is higher than in $\beta$-thalassemia intermedia due to the suppressive effect of transfusions on ineffective erythropoiesis ${ }^{19}$. Recently, it was shown that carriers of $\beta$ - or $\alpha$-thalassemia allele too, have altered iron metabolism parameters and erythropoiesis despite the absence of clinical symptoms ${ }^{20,22}$.

In this study, we analyzed for the first time, whether and how disrupted erythropoiesis in erythrocyte membrane defects, namely HS and HE, influences iron metabolism. The anemia in these patients was hyperchromic and milder than for thalassemia carriers. Due to chronic hemolysis it was accompanied by increased reticulocyte count. However, serum EPO was higher than in thalassemia carriers and together with significantly elevated sTfR, reflected increased erytropoietic activity in the bone marrow. In addition, slightly increased levels of GDF15 indicated some degree of ineffective erythropoiesis ${ }^{7}$.

The evaluation of parameters of iron metabolism revealed normal serum $\mathrm{Fe}$, but significantly elevated ferritin levels. As in thalassemia carriers, hepcidin and hepcidin/ ferritin ratio were reduced 1.7 and 3.7 times, respectively, compared to the controls. The patients with erythrocyte membrane defects also had a significantly lower (hepcidin/ferritin)/sTfR index than controls and dislocated from them on the graph when sTfR was plotted against hepcidin/ferritin. The erythrocyte membrane defects were shifted more to the top right on the graph than thalassemia carriers indicating more pronounced disruption in the erythropoiesis-hepcidin-iron stores axis. These results also suggest that hepcidin synthesis is inappropriately attenuated in patients with HS and HE. The direct signals need to be identified because hepcidin inhibition by EPO is indirect and requires active bone marrow erythropoiesis $^{13,23,24}$ and GDF15 is considered a marker of ineffective erythropoiesis rather than the main suppressor of hepcidin ${ }^{17}$. Very promising is the description of an erythroid regulator, named erythroferrone which stimulates hepcidin synthesis in response to chronic bleeding and EPO administration ${ }^{8}$. The human assay for erythroferrone measurements which would enable us to determine its levels under physiological and different pathological conditions, including erythrocyte membrane defects, is currently under development. 
We also showed that pediatric thalassemia carriers have altered $\mathrm{RBC}$ parameters; raised $\mathrm{RBC}$ count and reduced $\mathrm{Hb}$ level, MCV, MCH and MCHC. Elevated sTfR and GDF15 indicate increased but ineffective erythropoiesis in the bone marrow. Similar data were recently published for schoolchildren with $\beta$-thalassemia trait from Sri Lanka $^{22}$ and also for adult thalassemia carriers ${ }^{20}$. However, the study on adult thalassemia carriers showed that the $\beta$-thalassemia trait is associated with more profound negative effects on erythropoiesis than the $\alpha$-thalassemia trait $^{20}$. We were unable to make such a comparison because of only three $\alpha$-thalassemia carriers included in our cohort. Although their parameters were comparable with $\beta$-thalassemia carriers, it will be important to analyze larger numbers of children with $\alpha$-thalassemia trait to draw final conclusions. Alternatively, the clinical and laboratory difference between $\alpha$ - and $\beta$-thalassemia carriers may become more obvious over time. It is also likely that the difference reflects the heterogeneity at the molecular level; mutant alleles causing more profound imbalance in globin production would be associated with more severe alteration in erythropoiesis.

In the thalassemia carrier group, all individuals were iron replete with $\mathrm{sFe}$ and ferritin indistinguishable from healthy controls. However, they showed reduced hepcidin levels and reduced hepcidin/ferritin ratio (both parameters approximately twice). These results are consistent with the data obtained on pediatric $\beta$-thalassemia carriers from Sri Lanka ${ }^{22}$. On the other hand, Guimaraes et al. ${ }^{20}$ found that only adult $\alpha$-thalassemia carriers had significantly decreased hepcidin, while hepcidin of adult $\beta$-thalassemia carriers was insignificantly higher than in controls. Again, we cannot draw any conclusions due to the minimal number of $\alpha$-thalassemia carriers we analyzed. The differences between $\beta$-thalassemia carriers may again reflect differences between pediatric age and adulthood or may eventually be also influenced by variable severity of the different $\beta$-thalassemia alleles. Nevertheless, when the hepcidin/ferritin ratio was plotted against sTfR, our thalassemia carriers dislocate from the control group like the $\alpha$ - and $\beta$-thalassemia carriers in Guimaraes's study $^{20}$. This result is consistent with the altered erythropoiesis and iron homeostasis in the thalassemia trait.

The clinical picture of anemia and the hematological and biochemical laboratory findings of our patient with TM were in agreement with the published data on TM subjects, including inappropriate suppression of hepcidin with respect to the degree of iron overload ${ }^{18}$. The low hepcidin/ferritin ratio and elevated sTfR and GDF15 reflect increased ineffective erythropoiesis which signals higher iron demand leading to increased iron absorption and recycling. This is consistent with the finding that iron overload in TM is a result of regular transfusion therapy and disturbed hepcidin synthesis ${ }^{19}$.

\section{CONCLUSIONS}

Pediatric patients with erythrocyte membrane defect and thalassemia carriers showed alterations in erythro- poiesis and iron metabolism. The regulation of iron homeostasis appeared to be more disbalanced in erythrocyte membrane defects than in thalassemia carriers, but to a lesser extent than in the TM subject. We propose that the degree of alteration may reflect the severity of diseasecausing mutation. We confirmed the results of Guimarães et al. ${ }^{20}$, that the (hepcidin/ferritin)/sTfR ratio distinguishes thalassemia carriers from healthy controls and showed that it also differentiates patients with erythrocyte membrane defect. We propose that these individuals may be more susceptible to iron overload than the general population. As suggested for thalassemia carriers ${ }^{20}$, the (hepcidin/ferritin)/sTfR ratio can be used to follow up patients with erythrocyte membrane defects for evolution of the disease with a focus on alterations in erythropoietic activity and potential risk of iron overload. This ratio may also eventually help to make treatment choices including timing of splenectomy in patients with erythrocyte membrane defect and excessive hemolysis.

Acknowledgement: The work was supported by the Ministry of Health Czech Republic, grant num.: NT13587, MHo and DP were supported by Czech Science Foundation, grant num.: 15-13732S; MHo and ZZ were supported by the Internal Grant of Palacky University, grant num.: LF_2015_015.

Author contributions: LS: data collection and analysis, contributed to GDF15 measurements, manuscript writing; DH: measured the levels of hepcidin; ZZ: determined GDF-15 levels; MD performed Hb electrophoresis, Sanger sequencing and cryohemolysis tests; MHa: responsibility for the hepcidin assay; VM: collected the patients' material and provided clinical information; JV: performed statistical analyses; MHo, DP: study design, results interpretation; All authors contributed to the editing of the manuscript.

Conflict of interest statement: None declared.

\section{REFERENCES}

1. Weatherall DJ. Phenotype-genotype relationships in monogenic disease: lessons from the thalassaemias. Nat Rev Genet 2001;2(4):24555.

2. Piel FB, Weatherall DJ. The a-thalassemias. N Engl J Med 2014;371(20):1908-16.

3. An X, Mohandas N. Disorders of red cell membrane. Br J Haematol 2008;141(3):367-75.

4. Ganz T. Hepcidin in iron metabolism. Curr Opin Hematol 2004;11:251-4.

5. Ganz T and Nemeth E. Hepcidin and disorders of iron metabolism. Annu Rev Med 2011;62:347-60.

6. Tanno T, Bhanu NV, Oneal PA, Goh SH, Staker P, Lee YT, Moroney JW, Reed $\mathrm{CH}$, Luban NL, Wang RH, Eling TE, Childs R, Ganz T, Leitman SF, Fucharoen S, Miller JL. High levels of GDF15 in thalassemia suppress expression of the iron regulatory protein hepcidin. Nat Med 2007;13(9):1096-101.

7. Tanno T, Miller JL. Iron loading and overloading due to ineffective erythropoiesis. Adv Hematol 2010;2010:358283.

8. Kautz L, Jung G, Valore EV, Rivella S, Nemeth E, Ganz T. Identification of erythroferrone as an erythroid regulator of iron metabolism. Nat Genet 2014;46(7):678-84.

9. Finch C. Regulators of iron balance in humans. Blood 1994;84(6):1697-702. 
10. Nemeth E. Hepcidin in beta-thalassemia. Ann N Y Acad Sci 2010;1202:31-5.

11. Shalev H, Perez-Avraham G, Kapelushnik J, Levi I, Rabinovich A Swinkels DW, Brasse-Lagnel C, Tamary H. High levels of soluble serum hemojuvelin in patients with congenital dyserythropoietic anemia type I. Eur J Haematol 2013;90(1):31-6.

12. Mojzikova R, Koralkova P, Holub D, Zidova Z, Pospisilova D, Cermak J, Striezencova Laluhova Z, Indrak K, Sukova M, Partschova M, Kucerova J, Horvathova M, Divoky V. Iron status in patients with pyruvate kinase deficiency: neonatal hyperferritinaemia associated with a novel frameshift deletion in the PKLR gene (p.Arg518fs), and low hepcidin to ferritin ratios. Br J Haematol 2014;165(4):556-63.

13. Pospisilova D, Holub D, Zidova Z, Sulovska L, Houda J, Mihal V Hadacova I, Radova L, Dzubak P, Hajduch M, Divoky V, Horvathova M. Hepcidin levels in Diamond-Blackfan anemia reflect erythropoietic activity and transfusion dependency. Haematologica 2014:99(7):e118-21.

14. Zadrazil J, Horak P, Horcicka V, Zahalkova J, Strebl P, Hruby M. Endogenous erythropoietin levels and anemia in long-term renal transplant recipients. Kidney and Blood Pressure Research 2007:30(2):108-16.

15. Divoky V, Walczyskova S, Pospisilova D, Priwitzerova M, Takacova S, Kostelecka I, Divoka M, Rozmanova S, Jarosova M, Cermak J, Indrak K. [Rare forms of hereditary anaemia in the Czech and Slovak populations - $\beta$ - and $\delta \beta$-thalassaemia and unstable haemoglobin variants] Vnitr Lek 2005;51:886-93.

16. Tanno T, Noel P, Miller JL. Growth differentiation factor 15 in erythroid health and disease. Curr Opin Hematol 2010;17(3):184-90.
17. Ronzoni L, Sonzogni L, Duca L, Graziadei G, Cappellini MD, Ferru E. Growth Differentiation Factor 15 expression and regulation during erythroid differentiation in non-transfusion dependent thalassemia. Blood Cells Mol Dis 2015:54(1):26-8.

18. Origa R, Galanello R, Ganz T, Giagu N, Maccioni L, Faa G, Nemeth E. Liver iron concentrations and urinary hepcidin in beta-thalassemia. Haematologica 2007;92(5):583-8.

19. Pasricha SR, Frazer DM, Bowden DK, Anderson GJ. Transfusion suppresses erythropoiesis and increases hepcidin in adult patients with $\beta$-thalassemia major: a longitudinal study. Blood 2013;122(1):124-33.

20. Guimarães JS, Cominal JG, Silva-Pinto AC, Olbina G, Ginzburg YZ, Nandi V, Westerman M, Rivella S, de Souza AM. Altered erythropoiesis and iron metabolism in carriers of thalassemia. Eur J Haematol 2015;94(6):511-8.

21. Papanikolaou G, Tzilianos M, Christakis J, Bogdanos D, Tsimirika K, MacFarlane J, Goldberg YP, Sakellaropoulos N, Ganz T, Nemeth E. Hepcidin in iron overload disorders. Blood 2005;105(10):4103-5.

22. Jones $E$, Pasricha SR, Allen A, Evans P, Fisher CA, Wray $K$ Premawardhena A, Bandara D, Perera A, Webster C, Sturges P, Olivieri NF, St Pierre T, Armitage AE, Porter JB, Weatherall DJ, Drakesmith $\mathrm{H}$. Hepcidin is suppressed by erythropoiesis in hemoglobin $E$ $\beta$-thalassemia and $\beta$-thalassemia trait. Blood 2015;125(5):873-80.

23. Vokurka M, Krijt J, Sulc K, Necas E. Hepcidin mRNA levels in mouse liver respond to inhibition of erythropoiesis. Physiol Res 2006;55(6):667-74.

24. Pak M, Lopez MA, Gabayan V, Ganz T, Rivera S. Suppression of hepcidin during anemia requires erythropoietic activity. Blood 2006;108(12):3730-5. 